\title{
L'échographie endocavitaire dans les suppurations anales : le poids des mots, le choc des photos...
}

\author{
V. de PARADES (1), Isabelle ETIENNEY (1), CH. A. CUENOD (2), P. ATIENZA (1) \\ (1) Service de Proctologie Médico-Interventionnelle, Groupe hospitalier Diaconesses - Croix Saint Simon, Paris \\ (2) Service de Radiologie, Hôpital Européen Georges Pompidou, Paris
}

\section{Endoluminal ultrasound in anal suppurations: of words and images...}

\section{ABRÉVIATIONS / ABBREVIATIONS :}

$\begin{array}{ll}\text { EEA } & \text { échographie endo-anale } \\ \text { IRM } & \text { imagerie par résonance magnétique } \\ \text { AES } & \text { anal endosonography } \\ \text { MRI } & \text { magnetic resonance imaging }\end{array}$

RÉSUMÉ

Initialement destinée à explorer la prostate, l'échographie endocavitaire a été progressivement modifiée pour l'étude du tube digestif. Elle a notamment connu un essor considérable en proctologie ces dix dernières années car cette technique d'accès aisé, anodine, simple à réaliser et peu coûteuse, fournit une excellente image anatomique de la région anorectale. Elle a ainsi contribué à améliorer la prise en charge thérapeutique des suppurations anales car elle en fournit un bilan topographique précis (orifice primaire, trajet fistuleux principal, extensions secondaires et/ou collections) et car elle permet simultanément d'évaluer l'état de l'appareil sphinctérien. Une imagerie par résonance magnétique complémentaire peut parfois s'avérer utile pour compléter le bilan loco-régional de certaines suppurations complexes (trajet principal haut situé, collections profondes, tissus cicatriciels, etc.).

"Un chirurgien ignorant l'anatomie taille son patient comme un aveugle scie du bois» (Dicton du moyen âge. Trodel-Costedoat $\mathrm{K}$. La chirurgie au Moyen-Age ou le corps médical désarticulé. Moyen-Age 1999; 12 : 22-6).

\section{SUMMARY}

Originally dedicated to the exploration of prostate, endoluminal ultrasound has progressively been modified for the study of the digestive tract. In particular, it has increasingly been applied to proctology in the past decade because this technique, which is easy to access to, innocuous, simple to perform and costless, provides an excellent anatomical view of the anorectal region. Thus it has contributed to improve the therapeutic management of anal suppurations since it provides accurate topographical data (primary opening, main fistulous track, secondary extensions and/or collections) and because it allows for the evaluation of the sphincter apparatus at the same time. An additional magnetic resonance imaging can sometimes be useful in order to complete the locoregional evaluation of some complex suppurations (main fistulous track located in the upper zone, deep collections, scar tissues etc.).

"A surgeon who ignores anatomy cuts his patient like a blind man saws wood" (proverb from the Middle Ages. Trodel-Costedoat K. La chirurgie au Moyen-Age ou le corps médical désarticulé. Moyen-Age 1999; $12: 22-6)$.

\section{INTRODUCTION}

Initialement destinée à explorer la prostate, l'échographie endocavitaire est apparue dans les années 50 [1] et a été progressivement modifiée pour l'étude du tube digestif. Elle a notamment permis de fournir une image anatomique interprétable de l'appareil sphinctérien anal et des espaces cellulo-graisseux péri-anorectaux avec une résolution satisfaisante et une bonne définition. De fait, elle a connu, ces dix dernières d'années, un essor considérable en proctologie, favorisé par les insuffisances de l'examen clinique et des autres techniques d'imagerie [2]. En particulier, l'échographie endo-anale (EEA) a été largement sollicitée dans le cadre des suppurations anales, notamment les fistules anales crypto-glandulaires, où sa place est désormais bien établie.

\section{MATÉRIEL D'ÉCHOGRAPHIE}

Il existe divers types de sondes dont le choix repose surtout sur les habitudes de l'opérateur.

On dispose ainsi d'une part, de sondes rigides et d'autre part, d'échoendoscopes. Les sondes rigides ne comportent pas d'optique et sont introduites à l'aveugle dans le canal anal. Les échoendoscopes

Tirés à part : V. de PARADES, Groupe hospitalier Diaconesses, Croix Saint Simon, 18, rue du Sergent Bauchat, 75012 Paris (France).

Mots-clés : collection, défect sphinctérien, échographie endo-anale, imagerie par résonance magnétique, orifice primaire, trajet fistuleux. Key-words : anal endosonography, collection, fistulous track, magnetic resonance imagery, primary opening, sphincter disruption. 
souples comportent une optique et sont béquillables. Il faut également distinguer les sondes rotatives axiales des sondes linéaires. Les sondes rotatives fournissent des images transversales sur 270 à $360^{\circ}$ de circonférence, perpendiculaires à l'axe du transducteur et à l'axe du canal anal. Les sondes linéaires fournissent des images longitudinales sur un angle de 120 à $160^{\circ}$, parallèles à l'axe du transducteur.

L'extrémité de la plupart de ces sondes est recouverte par un ballonnet, rempli de 20 à $60 \mathrm{ml}$ d'eau dégazée, fournissant une interface acoustique entre le transducteur et la paroi anorectale. L'utilisation de ce ballonnet est optimale au niveau de l'ampoule rectale mais peut s'avérer difficile, voire désagréable, au niveau de l'anus. Pour pallier cet inconvénient, la firme danoise Bruel \& Kjaer a mis au point un cône rigide écho-transparent, rempli d'eau dégazée, de 10 ou $23 \mathrm{~mm}$ de diamètre, spécifiquement destiné à l'étude du canal anal. Ce matériel fournit des images d'excellente qualité et a largement contribué au développement de l'EEA.

La fréquence utilisée en pratique courante varie entre 5 et $10 \mathrm{MHz}$. Avec une sonde de $7,5 \mathrm{MHz}$, la profondeur de champ est d'environ $5 \mathrm{~cm}$ et le pouvoir de résolution axiale de l'ordre du millimètre [3].

\section{MODALITÉS D'EXAMEN}

L'examen est réalisé en ambulatoire après un lavement évacuateur rectal. Le patient est installé en position gynécologique ou en décubitus latéral. La sédation est le plus souvent inutile. Dans un premier temps, il est souhaitable de faire un examen proctologique sommaire afin d'établir une corrélation clinico-échographique et de limiter ainsi le risque de compte rendu discordant. Les images sont étudiées au fur et à mesure du retrait progressif de la sonde, et peuvent être imprimées et/ou enregistrées. La technique est simple et rapide à mettre en œuvre (environ 10 à 15 minutes), peu coûteuse (K30) et dénuée d'effets secondaires [3].

\section{IMAGES PATHOLOGIQUES}

L'EEA permet de faire le bilan topographique des suppurations anales (Tableau I) (schémas $n^{\circ} 1$ à 6 ) et d'évaluer l'appareil sphinctérien.

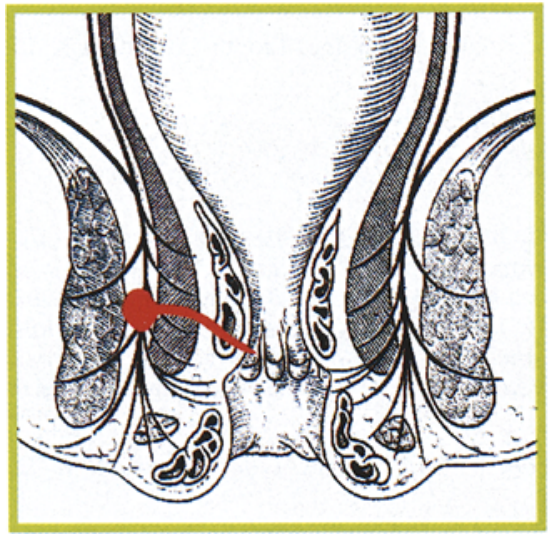

Figure 1

Orifice primaire cryptique

Cryptic primary opening

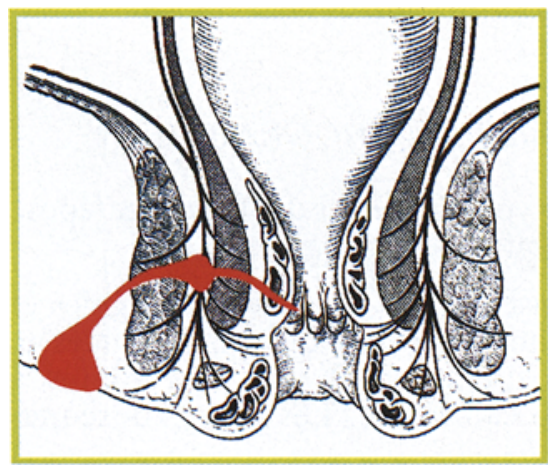

Figure 4

Trajet fistuleux trans-sphinctérien avec collection ischio-anale superficielle

Trans-sphincterian fistulous track with superficial ischio-anal collection

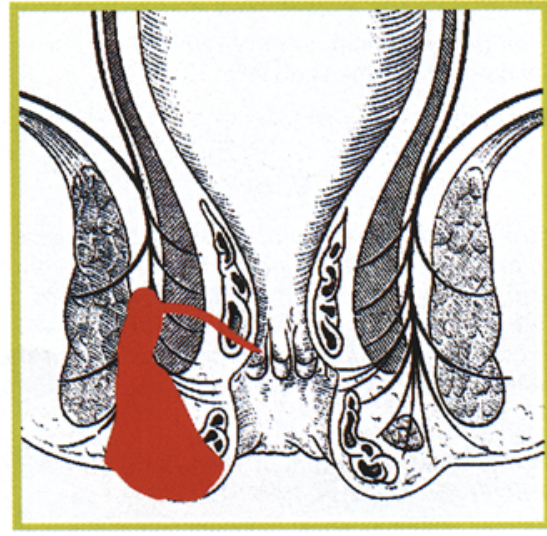

Figure 2

Trajet fistuleux intersphinctérien avec collection marginale

Fistulous track with marginal collection

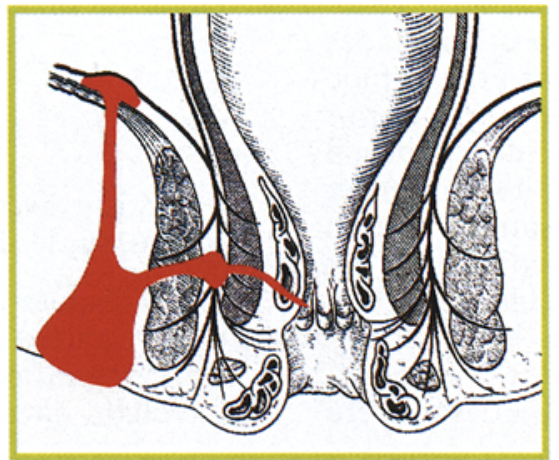

Figure 5

Trajet fistuleux trans-sphinctérien avec collection ischio-anale et extension supralévatorienne

Trans-sphincterian fistulous track with ischio-anal collection and supra-levatorian extension

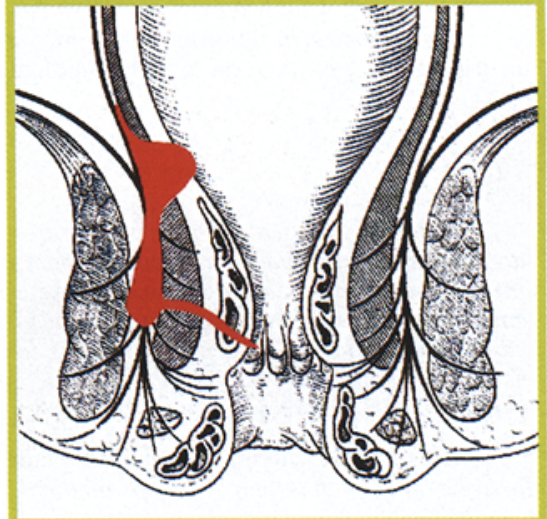

Figure 3

Trajet fistuleux intersphinctérien avec collection intramurale

Fistulous track with intra-mural collection

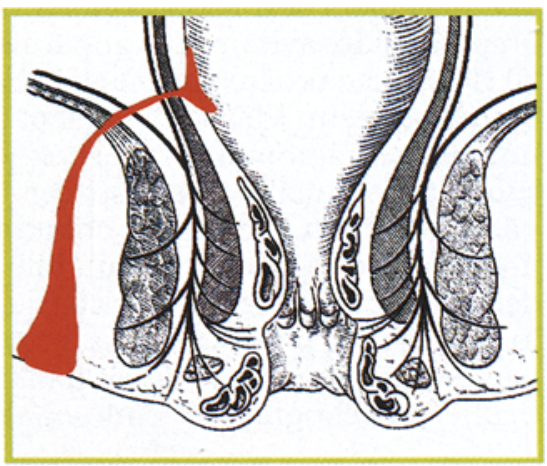

Figure 6

Trajet fistuleux extrasphinctérien Extra-sphincterian fistulous track 
TABLEAU I

CLASSIFICATION SIMPLIFIÉE DES FISTULES ANALES SELON LUNNISS et al. (44)

\begin{tabular}{|l|}
\hline Orifices : \\
\hline - primaire (cryptes anales, ulcérations rectales, etc.) \\
- secondaire (peau de la fesse ou de la marge anale, muqueuse \\
rectale ou vulvo-vaginale)
\end{tabular}

\section{Orifice primaire $\left(\right.$ photo $\left.n^{\circ} 1\right)$}

Il est repéré par une zone hétérogène siégeant au niveau de la première couche hyperéchogène (muqueuse) et/ou au niveau de la deuxième couche hypoéchogène (sphincter interne) [4]. D'éventuelles bulles d'air formant des images hyperéchogènes avec cône d'ombre postérieur caractéristique sont aisément reconnaissables et en facilitent le repérage [511]. Il est toutefois difficile d'affirmer que l'orifice primaire siège au niveau d'une crypte anale puisque la ligne pectinée n'est pas repérable en EEA [12].

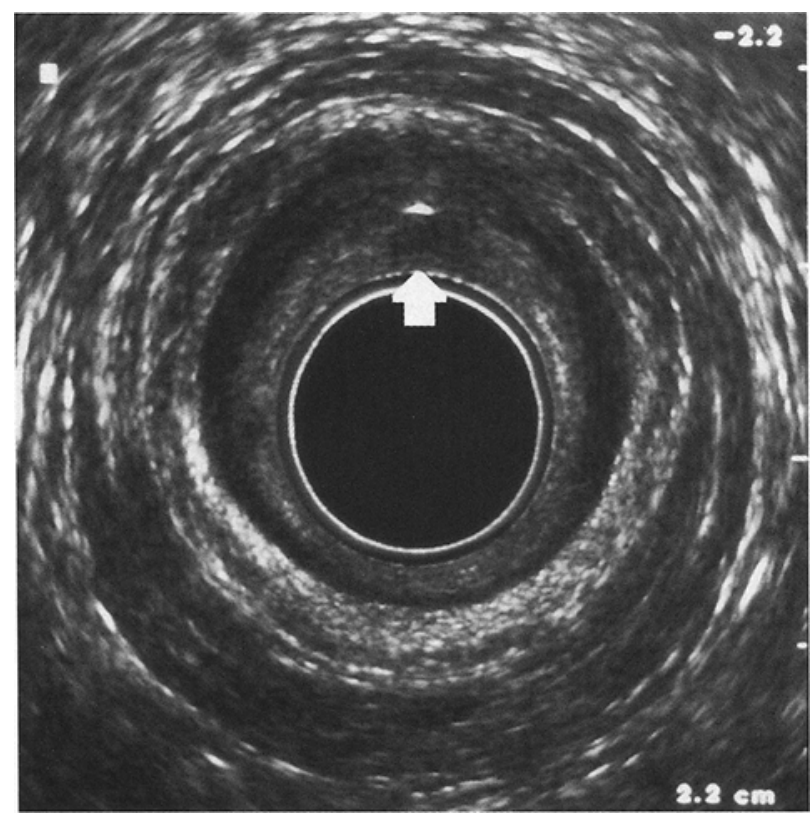

Photo 1

Orifice primaire antéro-médian (flèche) Antero-median primary opening (arrow)

\section{Trajet fistuleux principal et éventuelles extensions} secondaires (photos $n^{\circ} 2$ à 7 )

Les trajets fistuleux sont en général bien visibles et prennent l'aspect d'une bande hypoéchogène et mal limitée, parfois centrée par des bulles d'air, le plus souvent située entre les sphincters interne et externe (trajet intersphinctérien) ou se prolongeant à travers le sphincter externe vers la fosse ischio-anale (trajet trans-sphinctérien) $[5,6,8-11,13-15]$. L'épaisseur des

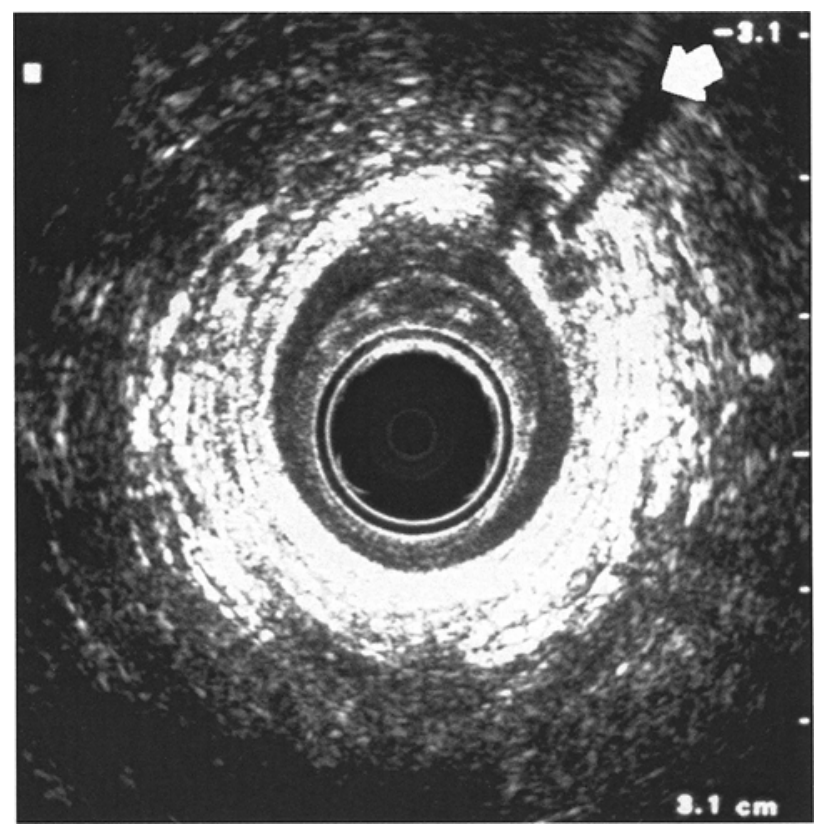

Photo 2

Trajet fistuleux trans-sphinctérien inférieur antéro-gauche (flèche) Antero-left lower trans-sphincterian fistulous track (arrow)

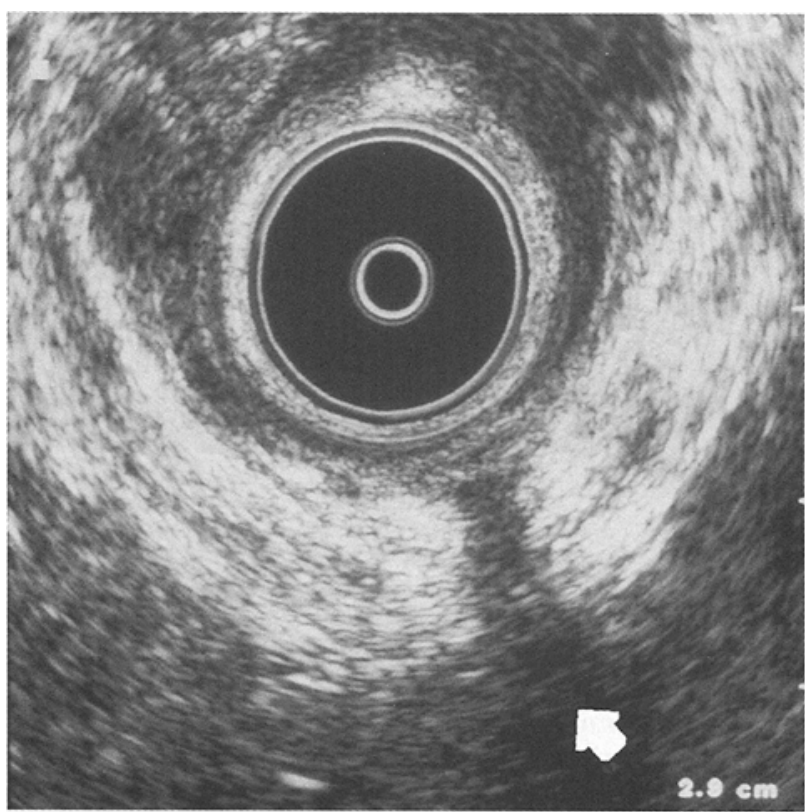

Photo 3

Trajet fistuleux trans-sphinctérien supérieur postéro-gauche (flèche) Postero-left upper trans-sphincterian fistulous track (arrow) 


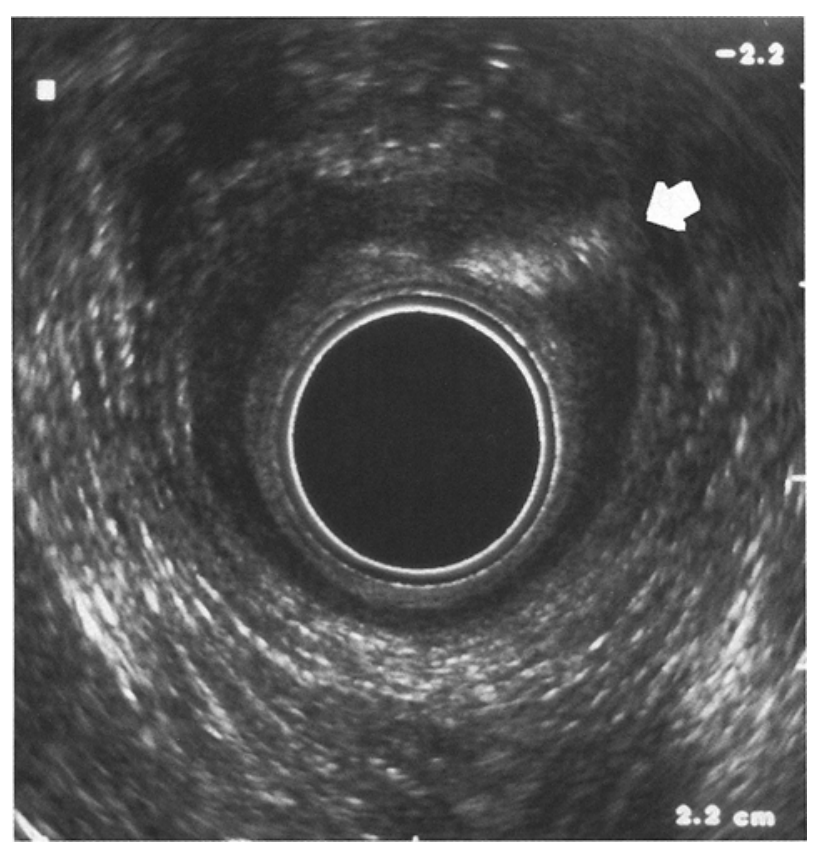

Photo 4

Trajet fistuleux trans-sphinctérien antéro-gauche hyperéchogène après injection d'air par l'orifice secondaire (flèche)

Hyperechogenous antero-left trans-sphincterian fistulous track after air insufflation through the secondary opening (arrow)

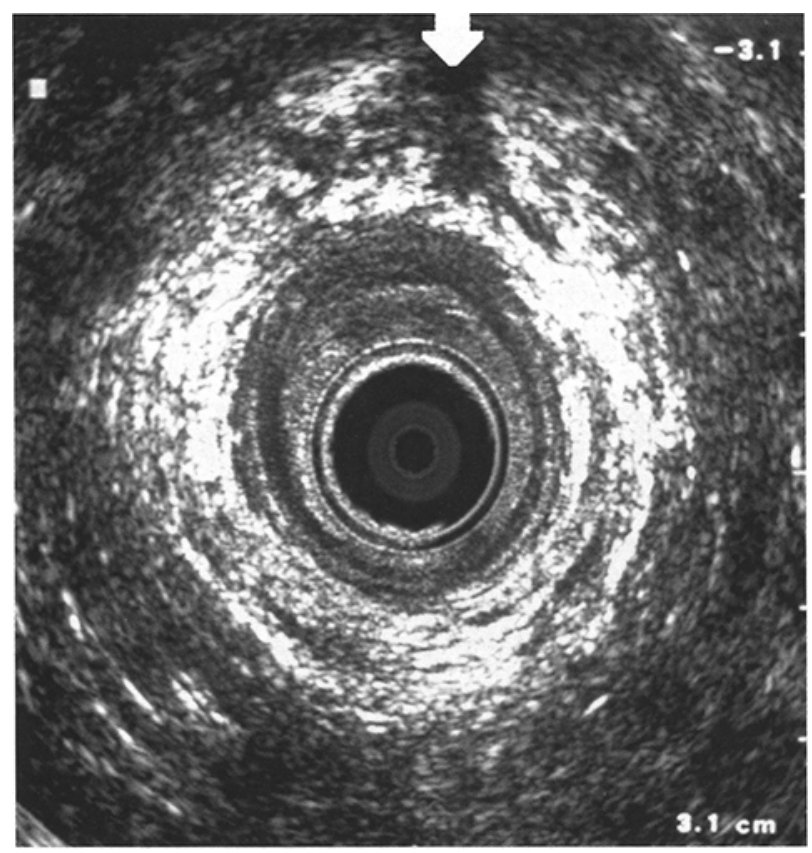

Photo 6

Trajet fistuleux trans-sphinctérien antérieur après anastomose iléo-anale (flèche)

Anterior trans-sphincterian fistulous track after ileo-anal anastomosis (arrow)

trajets est plus importante si l'examen est réalisé en période inflammatoire en raison de l'aspect hypoéchogène du pus sous tension et/ou de l'œdème périfistuleux. Les trajets extrasphinctériens (ne concernant pas l'appareil sphinctérien) sont plus difficiles à repérer car situés à distance du champ d'exploration de la sonde [6].

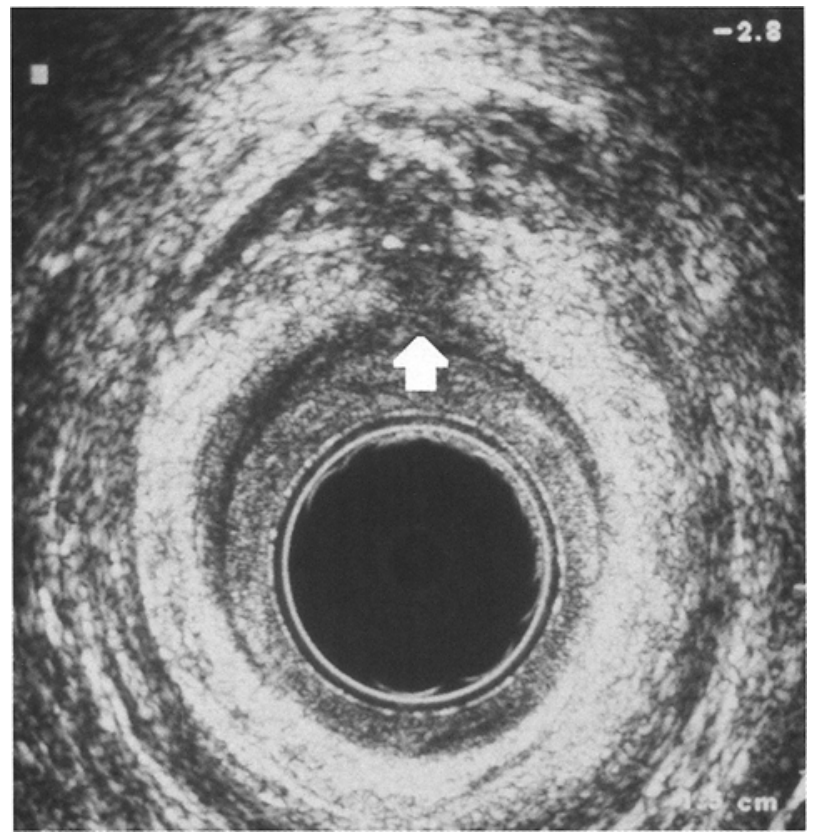

Photo 5

Trajet fistuleux trans-sphinctérien ano-vaginal (flèche) Ano-vaginal trans-sphincterian fistulous track (arrow)

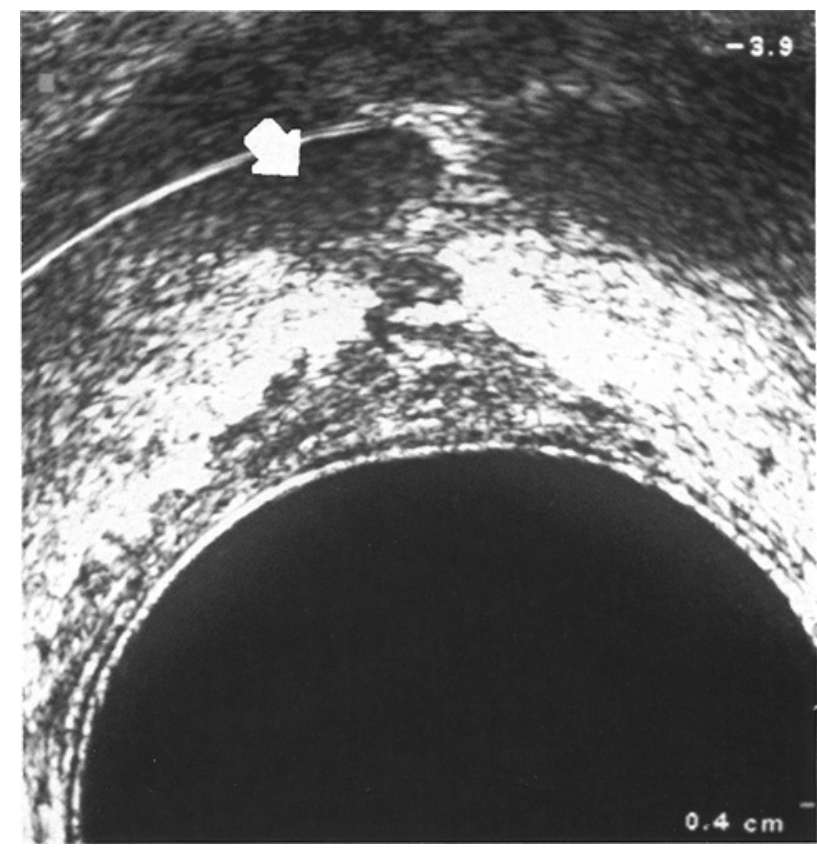

Photo 7

Trajet fistuleux recto-vésical (flèche) Recto-vesical fistulous track (arrow)

Les fistules trans-sphinctériennes anorecto-vaginales posent un problème spécifique. Les trajets anovaginaux sont en général aisément visualisés, a fortiori s'ils contiennent des bulles d'air et/ou s'ils sont inflammatoires. En revanche, les trajets recto-vaginaux sont difficiles à mettre en évidence, probablement en raison de leur longueur assez courte [16-21]. 


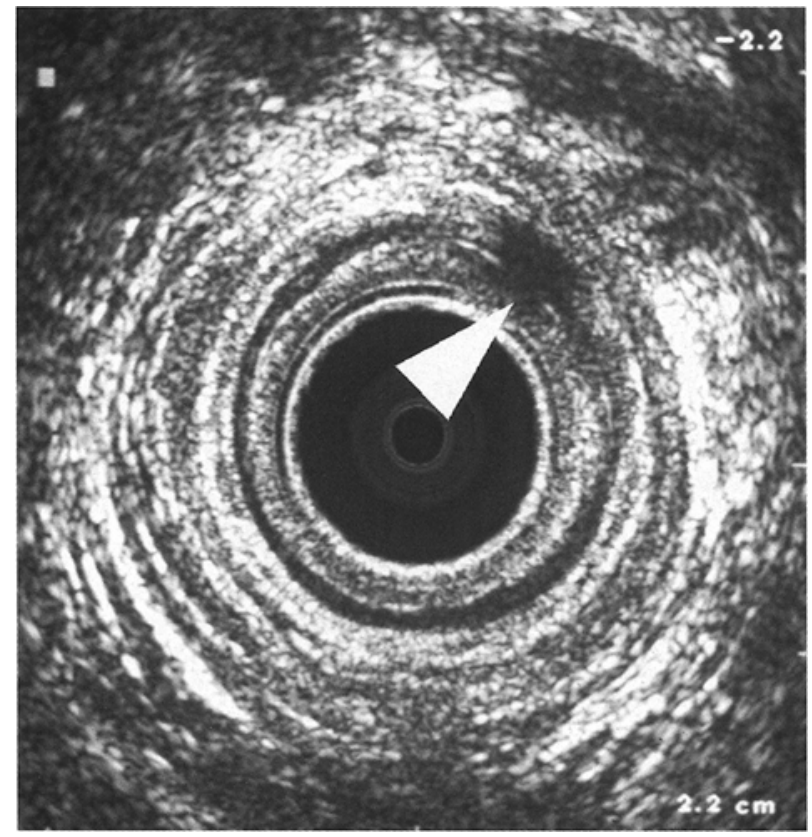

Photo 8

Collection intersphinctérienne antéro-gauche aiguë purulente (flèche) Purulent acute antero-left inter-sphincterian collection (arrow)

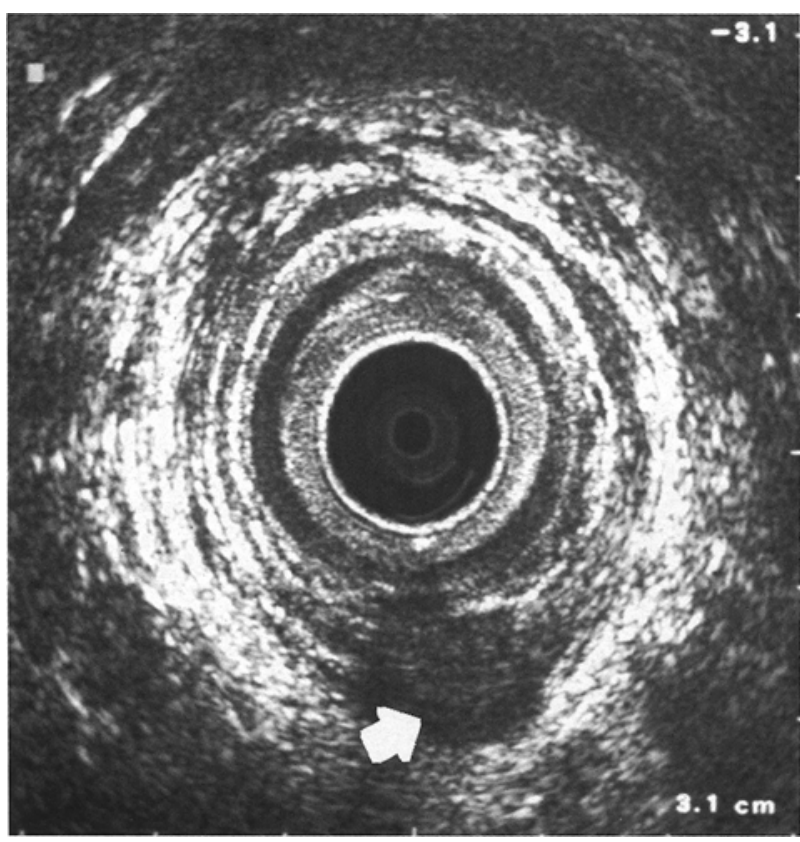

Photo 10

Collection intersphinctérienne sous-fissuraire postérieure (flèche) Posterior sub-fissurar inter-sphincterian collection (arrow)

\section{Collections (photos $n^{\circ} 8$ à 17 )}

Leur forme est variable et leur échogénicité dépend de leur contenu. Les collections purulentes, très inflammatoires, sont en général franchement hypoéchogènes et mal limitées du fait de l'œdème périphérique. Les collections chroniques, peu ou pas inflammatoires, contenant des granulations non

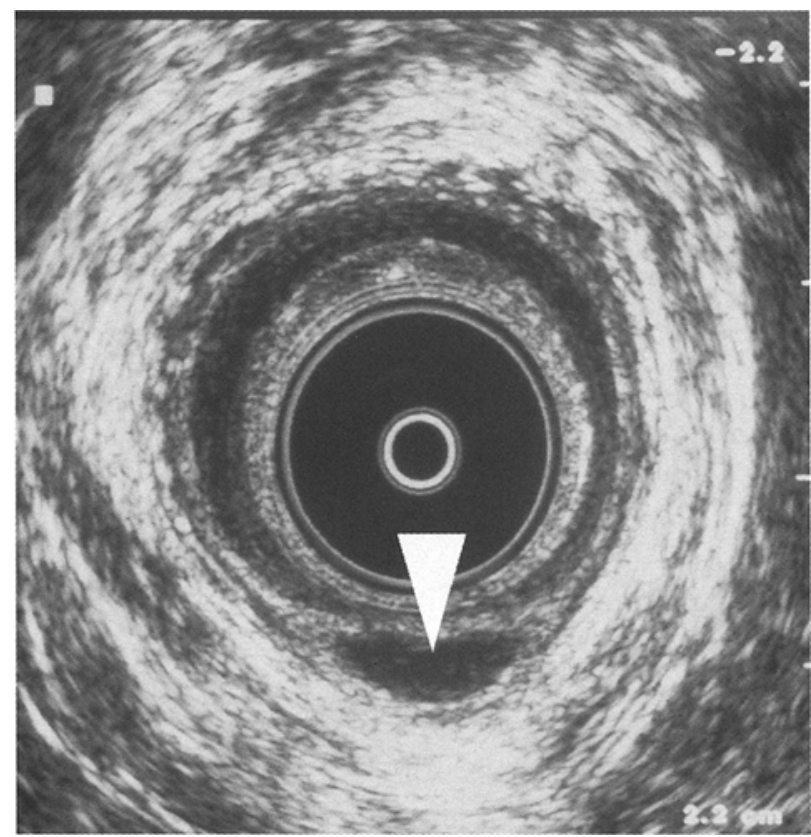

Photo 9

Collection intersphinctérienne postéro-médiane chronique (flèche) Chronic postero-median inter-sphincterian collection (arrow)

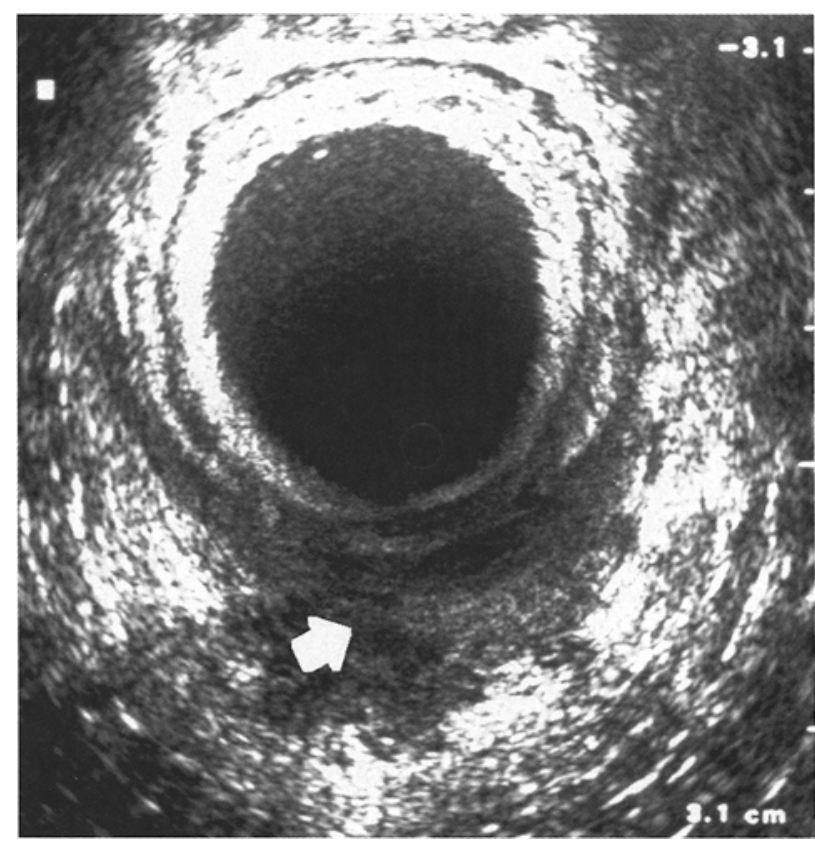

Photo 11

Collection intramurale postérieure (flèche) Posterior intra-mural collection (arrow)

purulentes et de la nécrose, sont hétérogènes et mieux limitées que les précédentes [5-8, 13-15]. Ces collections chroniques peuvent être difficiles à distinguer des tissus cicatriciels, particulièrement fréquents chez les patients multi-opérés et/ou dans le cadre de la maladie de Crohn, et cela constitue la limite principale de l'EEA $[5,6]$. 


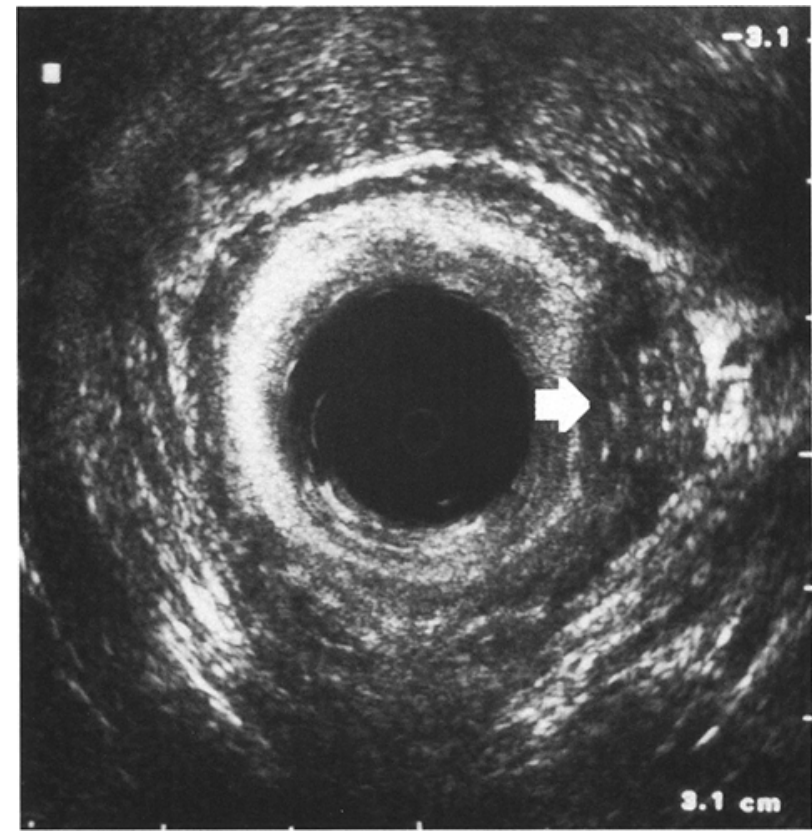

Photo 12

Collection intramurale latéro-gauche (flèche) sur corps étranger ingéré (cure-dents)

Latero-left intra-mural collection (arrow) after foreign material ingestion (toothpick)

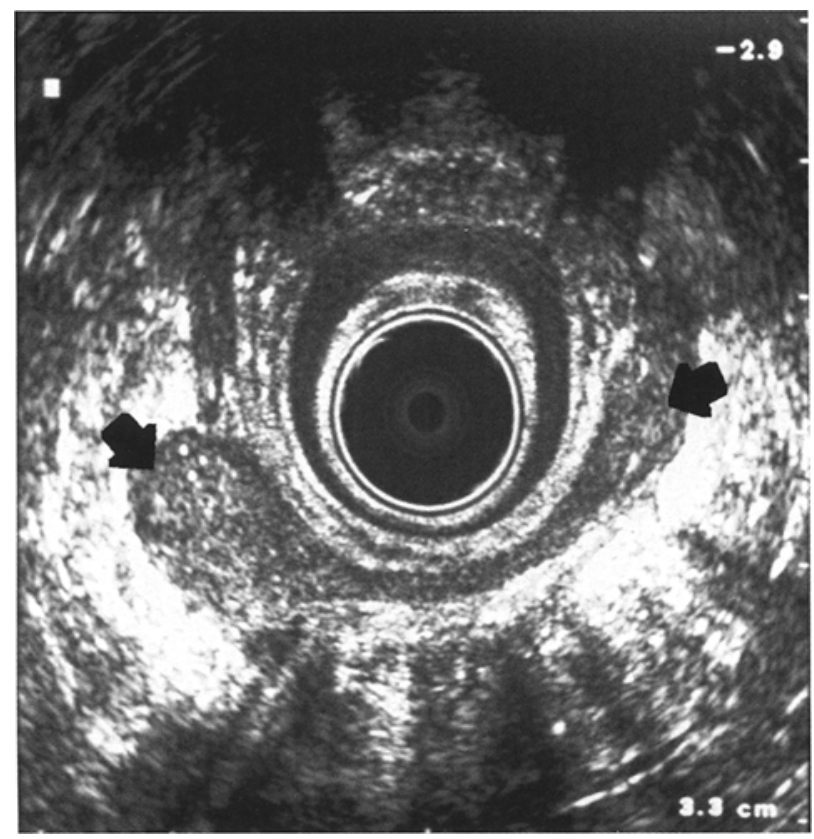

Photo 14

Collection ischio-anale en fer à cheval postérieur (flèches) Posterior horse shoe-shaped ischio-anal collection (arrow)

L'aspect échographique varie également selon les espaces anatomiques dans lesquels la suppuration a diffusé. Les collections intersphinctériennes et/ou intramurales sont particulièrement faciles à objectiver en EEA. En revanche, certaines collections ischio-anales profondes ou, surtout, supralévatoriennes peuvent être difficiles à visualiser car situées à distance de la sonde $[5,6]$.

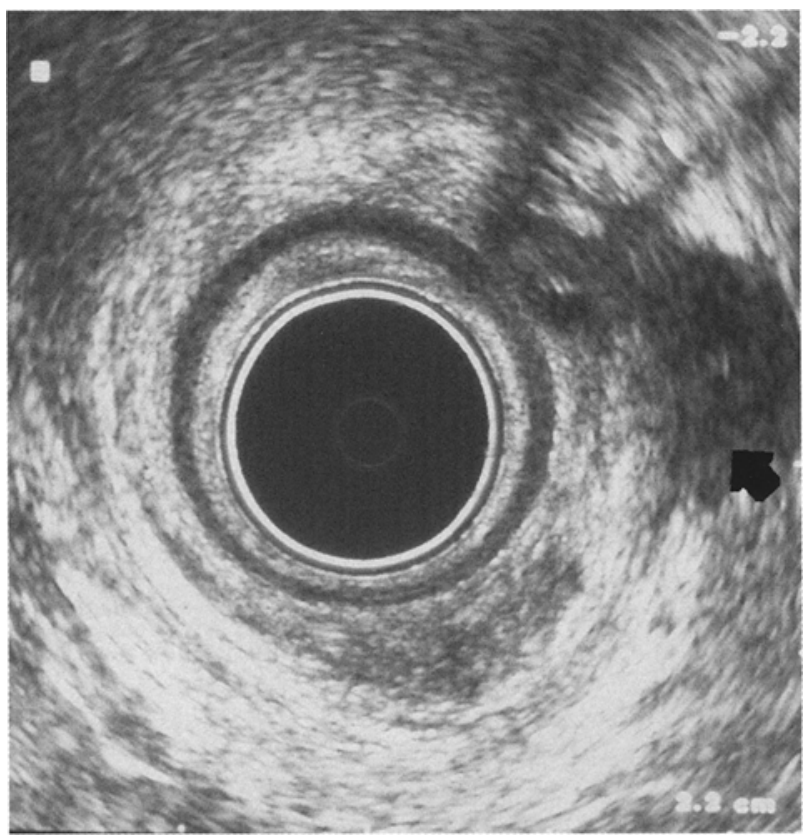

Photo 13

Collection ischio-anale latéro-gauche (flèche) Latero-left ischio-anal collection (arrow)

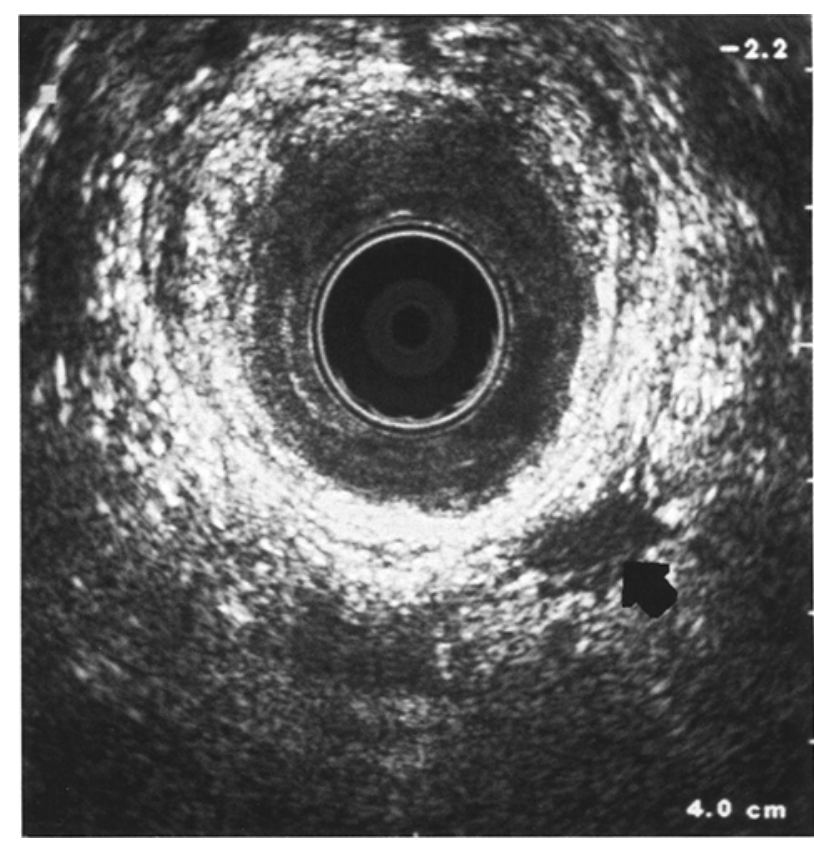

Photo 15

Collection supralévatorienne postéro-gauche (flèche) Posterio-left supra-levatorian collection (arrow)

Défects de l'appareil sphinctérien anal (photo ${ }^{\circ} 18$ )

L'EEA permet d'étudier l'appareil sphinctérien et d'en évaluer les défects éventuels. De tels défects sont fréquents en cas de suppuration multi-opérée. La sensibilité et la spécificité de l'EEA sont dans ce domaine proches de $100 \%$ [22-25]. Nous ne reviendrons pas davantage sur cette indication déjà étudiée antérieurement $[2,3,26]$. 


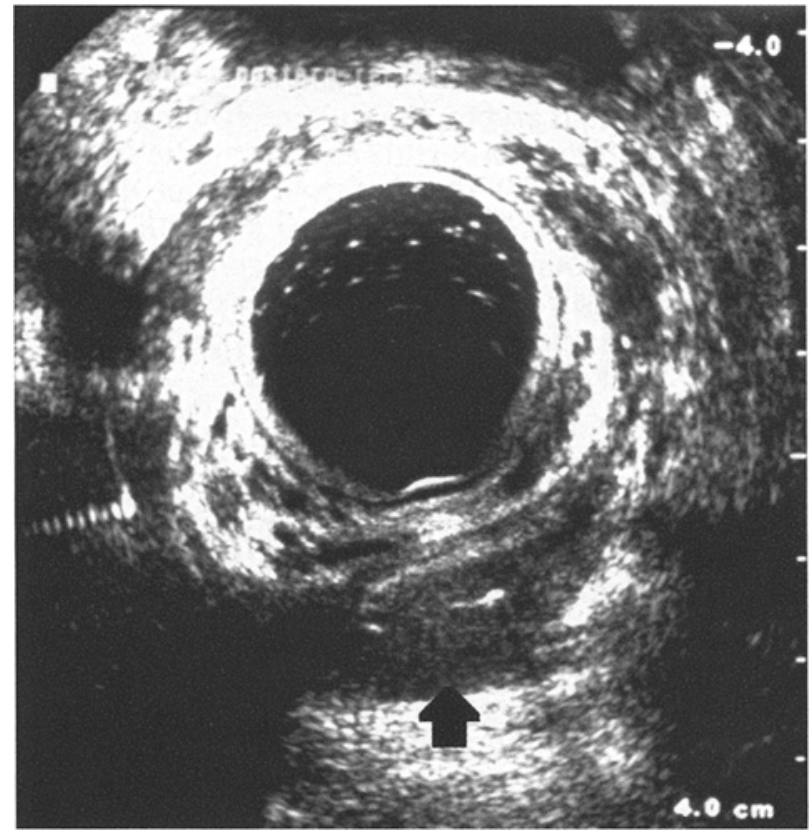

Photo 16

Collection pelvienne rétro-colique après anastomose colo-anale (flèche) Retro-colonic pelvic collection after colo-anal anastomosis (arrow)

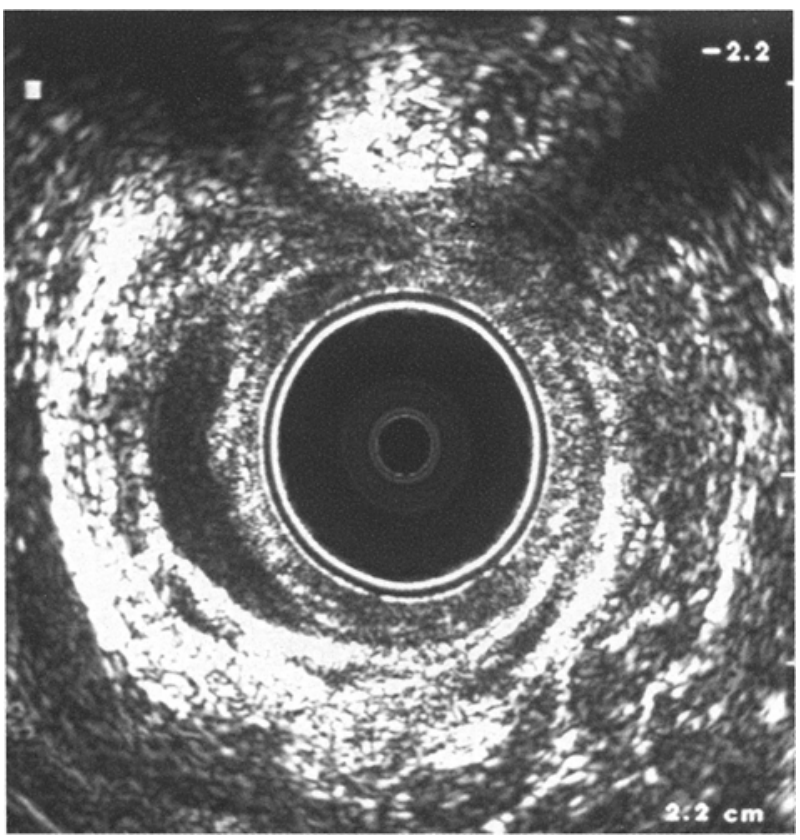

Photo 18

Défect latéro-gauche des sphincters interne et externe après chirurgie pour fistule anale

Latero-left defect of the internal and external sphincters after surgery for fistula in ano

\section{AMÉLIORATION DES PERFORMANCES DE L'ECHOGRAPHIE}

D'une façon générale, l'imagerie fournit des images plus franches lorsque la suppuration est active (présence de pus sous tension et d'œdème). Un exa-

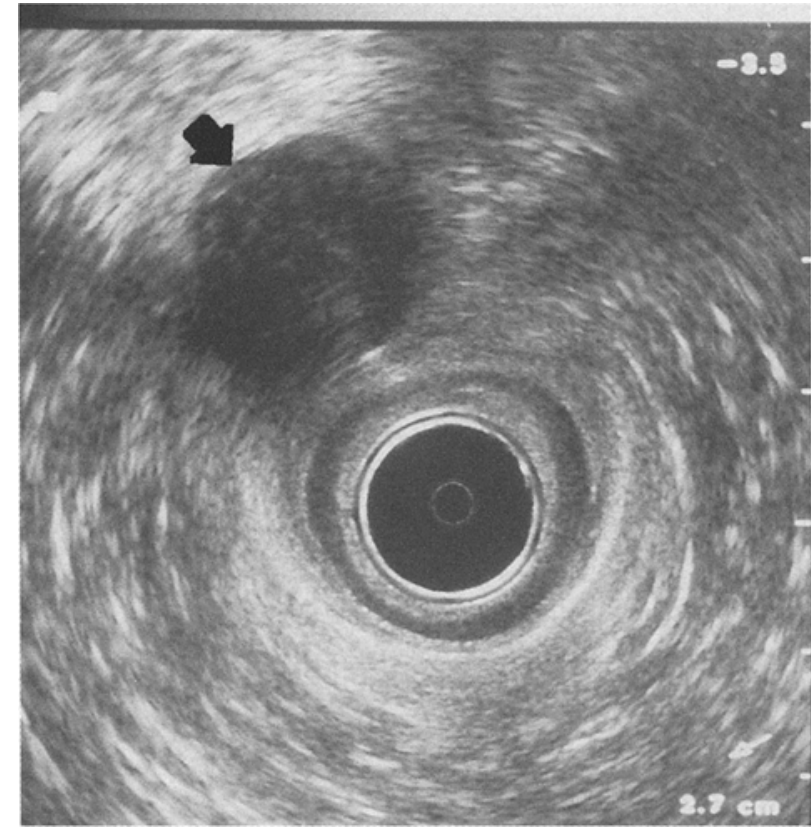

Photo 17

Bartholinite droite (flèche) Right bartholinitis (arrow)

men non contributif en phase quiescente chez un patient ayant une suppuration anale intermittente doit donc être répété en phase active, au moment de la récidive des douleurs [27-29].

Il est également possible de rehausser les images échographiques pathologiques par l'injection d'air ou mieux d'eau oxygénée dans le trajet fistuleux par le(s) éventuel(s) orifice(s) secondaire(s) [30]. Les trajets et les collections, initialement hypoéchogènes, deviennent ainsi hyperéchogènes et plus faciles à visualiser $[4,9-11,20,21,31-36]$. D'autres produits de contraste sont à l'étude [37].

Certains préconisent de compléter l'examen endoanal par la voie endovaginale [38].

Si la sonde le permet, il peut être utile de faire varier les fréquences. Par exemple, une fréquence de $10 \mathrm{MHz}$ est préférable pour l'identification de l'orifice primaire alors que $5 \mathrm{MHz}$ conviennent mieux à la recherche d'un trajet extrasphinctérien et/ou d'une collection supralévatorienne.

La localisation précise en hauteur de l'orifice primaire est difficile sur les images transversales obtenues avec les sondes rotatives axiales. Les sondes linéaires sectorielles, fournissant des images longitudinales, seraient plus performantes. En outre, ces sondes ont probablement plus de facilité à suivre les trajets fistuleux sur toute leur longueur [39].

L'EEA tridimensionnelle est encore récente et les données dont on dispose sont embryonnaires. On peut toutefois supposer qu'elle va contribuer à améliorer la mise en évidence des trajets fistuleux complexes $[12,40]$. 
Enfin, chez les patients hyperalgiques, la possibilité de déplacer la console d'échographie permet de réaliser l'examen au bloc opératoire, sous anesthésie, en préopératoire immédiat.

\section{PLACE AU SEIN \\ DES AUTRES EXPLORATIONS}

L'EEA est plus performante que l'examen clinique sous anesthésie $[6,8]$ et que la tomodensitométrie axiale transverse $[14,15]$ dans le bilan topographique des suppurations anales.

En revanche, elle est complémentaire de l'imagerie par résonance magnétique (IRM) [12]. L'EEA est en effet plus facile d'accès, plus simple à mettre en œuvre et moins coûteuse. De plus, elle serait meilleure pour l'évaluation de l'appareil sphinctérien anal [41-43] ainsi que pour la mise en évidence des orifices primaires et des collections intersphinctériennes et/ou intramurales [27, 39, 44-47]. Cela étant dit, l'IRM fournit des images d'une excellente qualité en raison de sa bonne résolution en contraste, en raison de sa possibilité de réaliser des coupes dans tous les plans de l'espace permettant une analyse tridimensionnelle, très intéressante en cas de suppuration complexe, et en raison de son excellente analyse à distance des espaces celluleux péri-anorectaux permettant un repérage aisé des collections ischio-anales et/ou supralévatoriennes. L'IRM peut également discriminer les tissus cicatriciels des collections et ainsi pallier une limite importante de l'EEA [5, 6, 27, 44, 48]. Enfin, elle est appelée à se perfectionner dans les années à venir en raison des nombreuses séquences possibles $[2,12,49,50]$. Il faut toutefois souligner que, de même que l'EEA est une technique opérateurdépendante, l'IRM nécessite une compétence spécifique pour sa réalisation et son interprétation si bien que le choix d'un praticien ayant une excellente connaissance de l'anatomie anorectale et des suppurations anales est essentiel [29].

Enfin, l'EEA n'a pas été comparée à la fistulographie mais cette technique est actuellement quasiment abandonnée $[2,12,51]$.

\section{INDICATIONS}

L'imagerie s'avère inutile dans la grande majorité des suppurations dont le traitement est d'emblée envisagé après le simple examen clinique. Toutefois, l'EEA peut être intéressante dans les situations suivantes:

\section{Doute diagnostique}

L'EEA permet d'étayer le diagnostic différentiel entre une fistule crypto-glandulaire et une suppuration non fistuleuse (bartholinite, maladie de Verneuil, sinus pilonidal infecté, furoncle, plaie d'épisiotomie infectée, etc.). Elle est aussi particulièrement performante pour rechercher une éventuelle suppuration sous-fissuraire. Enfin, en cas de douleurs inexpliquées, elle peut révéler une suppuration non détectée par l'examen clinique $[12,52]$.

\section{Suppuration complexe}

Une suppuration complexe se caractérise par son extension anatomique inhabituelle (trajet principal haut situé, extensions secondaires multiples et/ou collections profondes), son évolution récidivante, ses traitements chirurgicaux multiples, les remaniements cicatriciels importants, une maladie de Crohn anopérinéale sous-jacente, etc. Un bilan topographique précis est alors essentiel afin d'optimiser l'efficacité du traitement. Une erreur d'identification d'orifice primaire ou la méconnaissance d'une collection profonde et/ou d'une extension secondaire pourrait en effet se solder par une absence de guérison et/ou par une récidive. Dans cette indication, l'EEA devrait être idéalement réalisée de concert avec l'IRM $[2,12$, $29,52,53]$.

\section{Crainte de survenue ou de majoration de troubles de la continence anale postopératoires}

La mise en évidence de défect(s) sphinctérien(s) a un intérêt médico-légal, notamment chez les patients multi-opérés. En outre, elle peut amener à privilégier une technique d'épargne sphinctérienne (drainage prolongé par séton non serré, abaissement d'un lambeau de recouvrement sur l'orifice primaire, injection de colle biologique et/ou anti-TNF alpha en cas de maladie de Crohn) plutôt qu'une classique fistulotomie susceptible d'altérer la continence anale $[9,12$, 29, 54].

\section{CONCLUSION}

D'utilisation récente, l'EEA s'est imposée comme une exploration complémentaire de référence en proctologie. Elle a notamment contribué à améliorer la prise en charge thérapeutique des suppurations car elle fournit un bilan topographique fiable des lésions et permet simultanément d'évaluer l'état de l'appareil sphinctérien. Cependant, une IRM complémentaire peut s'avérer parfois utile pour pallier ses limites, particulièrement dans les cas de suppurations complexes. 
1. Wild JJ. The use of ultrasonic pulses for the measurements of biologic tissues and the detection of tissue density changes. Surgery $1950 ; 27: 183-8$.

2. Stoker J, Rociu E, Wiersma TG, Lameris JS. Imaging of anorectal disease. Br J Surg 2000; 87 : 10-27.

3. Damon H, Henry L, Valette PJ, Mion F. Apport de l'échographie endo-anale dans les affections proctologiques non tumorales. Gastroentérol Clin Biol 2001 ; 25 : 35-44.

4. Cho DY. Endosonographic criteria for an internal opening of fistula-in-ano. Dis Colon Rectum $1999 ; 42: 515-8$

5. Law PJ, Talbot RW, Bartram CI, Northover JM. Anal endosonography in the evaluation of perineal sepsis and fistula in ano. Br J Surg 1989; $76: 752-5$.

6. Choen S, Burnett S, Bartram CI, Nicholls RJ. Comparaison between anal endosonography and digital examination in the evaluation of anal fistulae. Br J Surg 1991; 78: 445-7.

7. Cataldo PA, Senagore A, Luchtefeld MA. Intrarectal ultrasound in the evaluation of perirectal abcesses. Dis Colon Rectum $1993 ; 36: 554-8$.

8. Deen KI, Williams JG, Hutchinson R, Keighley MR, Kumar D. Fistulas in ano : endoanal ultrasonographic assessments assists decision making for surgery. Gut $1994 ; 35: 391-4$.

9. Lindsey I, Humphreys MM, George BD, Mortensen NJ. The role of anal ultrasound in the management of anal fistulas. Colorectal Dis 2002; $4: 118-22$.

10. Sudol-Szopinska I, Gesla J, Jakubowski W, Noszczyk W, Szczepkowsi M, Sarti D. Reliability of endosonography in evaluation of anal fistulae and abscesses. Acta Radiol. 2002; 43 : 599. 602 .

11. Lengyel AJ, Hurst NG, Williams JG. Pre-operative assessment of anal fistulas using endoanal ultrasound. Colorectal Dis $2002 ; 4: 436-40$.

12. Bartram $C$, Buchanan $G$. Imaging anal fistula. Radiol Clin North Am 2003; $41: 443-57$.

13. Tio TL, Mulder CJ, Wijers OB, Sars PR, Tytgat GN. Endosonography of peri-anal and peri-colorectal fistula and/or abcess in Crohn's disease. Gastrointest Endosc 1990; 36 : 331-6.

14. Van Outryve MJ, Pelckmans PA, Michielsen PP, Van Maercke YM. Value of transrectal ultrasonography in Crohn's disease. Gastroenterology 1991; 101:1171-7.

15. Schratter-Sehn AU, Lochs $H$, Vogelsang $H$, Schurawittzki $H$, Herold C, Schratter M. Endoscopic ultrasonography versus computed tomography in the differential diagnosis of perianorectal complications in Crohn's disease. Endoscopy 1993; 25 : 582-6.

16. Tsang CB, Madoff RD, Wong WD, Rothenberger DA, Finne $\mathrm{CO}$, Singer D, Lowry AC. Anal sphincter integrity and function influences outcome in rectovaginal fistula repair. Dis Colon Rectum 1998; 41 : 1141-6.

17. Yee L, Birnbaum EH, Read TE, Kodner IJ, Fleshman JW. Use of endoanal ultrasound in patients with rectovaginal fistulas. Dis Colon Rectum 1999 ; 42 : 1057-64.

18. Baig MK, Zhao RH, Yuen CH, Nogueras JJ, Singh JJ, Weiss $\mathrm{EG}$, Wexner SD. Simple rectovaginal fistulas. Int J Colorect Dis $2000 ; 15: 323-7$.

19. Stoker J, Rociu E, Schouten WR, Lameris JS. Anovaginal and rectovaginal fistulas : endoluminal sonography versus endoluminal MR imaging. AJR 2002; 178:737-41.

20. Sudol-Szopinska I, Jakubowski W, Szczepkowski M. Contrastenhanced endosonography for the diagnosis of anal and anovaginal fistulas. J Clin Ultrasound 2002; 30 : 145-50.

21. Sudol-Szopinska I, Jakubowski W, Szczepkowski M, Sarti D. Usefulness of hydrogen peroxide enhancement in diagnosis of anal and ano-vaginal fistulas. Eur Radiol 2003; $13: 1080-4$

22. Deen KI, Kumar D, Williams JG, Olliff J, Keighley MR. Anal sphincter defects. Correlation between endoanal ultrasound and surgery. Ann Surg 1993; $218: 201-5$.

23. Sultan AH, Kamm MA, Talbot IC, Nicholls RJ, Bartram CI. Anal endosonography for identifying external sphincter defects confirmed histologically. Br J Surg 1994; 81 : 463-5.
24. Meyenberger C, Bertschinger P, Zala GF, Buchmann P. Anal sphincter defects in fecal incontinence : correlation between endosonography and surgery. Endoscopy $1996 ; 28: 217-24$.

25. Romano G, Rotondano G, Esposito P, Pellecchia L, Novi A. External anal sphincter defects : correlation between pre-operative anal endosonography and intraoperative findings. $\mathrm{Br} \mathrm{J}$ Radiol 1996; $69: 6-9$.

26. de Parades V, Etienney I, Atienza P. Les défects sphinctériens anaux en endosonographie. Acta Endoscopica $2002 ; 32: 15-29$.

27. Hussain SM, Stoker J, Schouten WR, Hop WC, Lameris JS. Fistula in ano: endoanal sonography versus endoanal MR imaging in classification. Radiology $1996 ; 200: 475-81$.

28. Stoker J, Rociu E, Zwamborn AW, Schouten WR, Lameris JS. Endoluminal MR imaging of the rectum and anus : technique, applications, and pitfalls. Radiographics $1999 ; 19: 383-8$.

29. de Parades V, Cuenod CA, Thomas C, Marteau P, Parisot C, Atienza P. L'imagerie dans la maladie de Crohn ano-périnéale. Acta Endoscopica $2000 ; 30: 565-77$.

30. Kruskal JB, Kane RA, Morrin MM. Peroxide-enhanced anal endosonography : technique, image interpretation, and clinical applications. Radiographics 2001; 21 : S173-S189.

31. Cheong DM, Nogueras JJ, Wexner SD, Jagelman DG. Anal endosonography for recurrent anal fistulas: image enhancement with hydrogen peroxyde. Dis Colon Rectum 1993; 36 : 1158-60.

32. Poen AC, Felt-Bersma RJ, Eijsbouts QA, Cuesta MA, Meuwissen SG. Hydrogen peroxide-enhanced transanal ultrasound in the assessment of fistula-in-ano. Dis Colon Rectum 1998; $41: 1147-52$.

33. Ratto C, Gentile E, Merico M, Spinazzola C, Mangini G, Sofo L, Doglietto $G$. How can the assessment of fistula-in-ano be improved? Dis Colon Rectum 2000; 43 : 1375-82.

34. Sloots CE, Felt-Bersma RJ, Poen AC, Cuesta MA. Assessment and classification of never operated and recurrent cryptoglandular fistulas-in-ano using hydrogen peroxide enhanced transanal ultrasound. Colorectal Dis $2001 ; 3: 422-6$.

35. Ortiz H, Marzo J, Jimenez G, DeMiguel M. Accuracy of hydrogen peroxide-enhanced ultrasound in the identification of internal openings of anal fistulas. Colorectal Dis 2002; 4 : 280-3.

36. Sloots CE, Felt-Bersma RJ, Poen AC, Cuesta MA, Meuwissen SG. Assessment and classification of fistula-in-ano in patients with Crohn's disease by hydrogen peroxyde enhanced transanal ultrasound. Int J Colorectal Dis 2001; $16: 292-7$.

37. Chew SS, Yang JL, Newstead GL, Douglas PR. Anal fistula : Levovist ${ }^{(-)}$-enhanced endoanal ultrasound. A pilot study. Dis Colon Rectum $2003 ; 46: 377-84$.

38. Poen AC, Felt-Bersma RJ, Cuesta MA, Meuwissen SG. Vaginal endosonography of the anal sphincter complex is important in the assessment of faecal incontinence and perianal sepsis. Br J Surg 1998; 85 : 359-63.

39. Orsoni P, Barthet M, Portier F, Panuel M, Desjeux A, Grimaud JC. Prospective comparison of endosonography, magnetic resonance imaging and surgical findings in anorectal fistula and abscess complicating Crohn's disease. Br J Surg 1999; 86 : 360-4.

40. West RL, Zimmerman DDE, Dwarkasing S, Hussain SM, Hop WCJ, Schouten WR, Kuipers EJ, Felt-Bersma RJF. Prospective comparison of hydrogen peroxide-enhanced three-dimensional endoanal ultrasonography and endoanal magnetic resonance imaging of perianal fistulas. Dis Colon Rectum 2003 ; $46: 1407-15$.

41. Rociu E, Stoker J, Eijkemans MJ, Schouten WR, Lameris JS. Fecal incontinence : endo-anal US versus endoanal MR imaging. Radiology $1999 ; 212: 453-8$.

42. Malouf AJ, Williams AB, Halligan S, Bartram CI, Dhillon S, Kamm MA. Prospective assesment of accuracy of endoanal MR imaging an endosonography in patients with fecal incontinence. AJR 2000; $175: 741-5$.

43. Matsuoka H, Desai MB, Wexner SD, Adami C, Mavrantonis C, Nogueras JJ, Weiss EG, Billotti VL, Nakamura T. A pilot assessment of whether external coil MRI is useful to assess evacuatory disorders. Int J Colorectal Dis $2000 ; 15: 91-5$. 
44. Lunniss PJ, Barker PG, Sultan AH, Armstrong P, Reznek RH, Bartram CI, Cottam KS, Phillips RK. Magnetic resonance imaging of fistula-in-ano. Dis Colon Rectum 1994; 37 : 708-18.

45. Maier AG, Funovics MA, Kreuzer SH, Herbst F, Wunderlich M, Teleky BK, Mittlbock M, Schima W, Lechner GL. Evaluation of perianal sepsis : comparison of anal endosnography and magnetic resonance imaging. J Magn Reson Imaging 2001 ; 14 : 254-60.

46. Gustafsson UM, Kahvecioglu B, Aström G, Ahlström H, Graf W. Endoanal ultrasound or magnetic resonance imaging for preoperative assessment of anal fistula : a comparative study. Colorectal Dis 2001 ; 3 : 189-97.

47. Schwartz DA, Wiersema MJ, Dudiak KM, Fletcher JG, Clain JE, Tremaine WJ, Zinsmeister AR, Norton ID, Boardman LA, Devine RM, Wolff BG, Young-Fadok TM, Diehl NN, Pemberton JH, Sandborn WJ. A comparison of endoscopic ultrasound, magnetic resonance imaging, and exam under anesthesia for evaluation of Crohn's perianal fistulas. Gas troenterology $2001 ; 121: 1064-72$.

48. Beckingham IJ, Spencer JA, Ward J, Dyke JW, Adams C, Ambrose NS. Prospective evaluation of dynamic contrast

\section{INTRODUCTION}

Originally dedicated to the exploration of prostate, endoluminal ultrasound appeared in the fifties [1] and has progressively been modified for the study of the digestive tract. Especially, it allowed to give an anatomical image of the anal sphincter apparatus and of the peri-anorectal cellular and adipose spaces that could be interpreted with a satisfactory resolution and a good definition. Accordingly, it has increasingly been applied to proctology in the past decade, favoured by the deficiencies of clinical examination and of the other imaging techniques [2]. In particular, anal endosonography (AES) has largely been used for fistulae in ano, especially crypto-glandular fistulae where its value is now well established.

\section{ULTRASOUND MATERIAL}

There are different types of probes for which the choice mostly relies upon the preference of the operator. On one hand, there are rigid probes, and on the other hand, the ultrasound endoscopes. The rigid probes do not have lenses and are blindly introduced into the anal canal. The flexible ultrasound endoscopes have an optical lens. One must also distinguish between axial rotative probes from linear ones. Rotative probes provide transversal images over 270 to $360^{\circ}$ of circumference, perpendicular to the axis of the transducer and to the axis of the anal canal. Linear probes provide longitudinal images over an angle of 120 to $160^{\circ}$, parallel to the axis of the transducer. The extremity of most of these probes is covered with a balloon, filled with 20 to $60 \mathrm{ml}$ of degassed water, providing an acoustic interface between the transducer and the anorectal wall. The usefulness of this balloon is optimal at the level of the rectal ampulla but can become poor, or even create discomfort, at the level of enhanced magnetic resonance imaging in the evaluation of fistula in ano. Br J Surg 1996; $83: 1396-8$.

49. Hussain SM, Outwater EK, Joekes EC, Ulrich F, Delemarre HB, Bemelman WA, Li X, Mitchell DG. Clinical and MR imaging features of cryptoglandular and Crohn's fistulas and abscesses. Abdom Imaging 2000; $25: 67-74$.

50. Cuenod CA, de Parades V, Siauve N, Marteau P, Grataloup C, Hernigou A, Berger A, Cugnenc PH, Frija G. IRM des suppurations ano-périnéales. J Radiol $2003 ; 84: 516-28$.

51. Kuijpers HC, Schulpen T. Fistulography for fistula-in-ano. Is it useful? Dis Colon Rectum 1985;28:103-4.

52. Williams JG. Anal ultrasonography in the diagnosis and management of abscess and fistula disease of the anorectum. Semin Colon Rectal Surg 1995; 6 : 105-13.

53. Wexner SD, Rosen L, Roberts PL, Lowry A, Burnstein M, Hicks T. Practice parameters for treatment of fistula-in-ano. Supporting documentation. Dis Colon Rectum 1996; 39 : 1363-72.

54. Garcia-Aguilar J, Belmonte C, Wong WD, Goldberg SM, Madoff RD. Anal fistula surgery : factors associated with recurrence and incontinence. Dis Colon Rectum 1996; 39 : 723-9.

the anus. In order to counter this problem, the Danish company Bruel \& Kjaer made an echo-clear rigid cone, filled with degassed water, from 10 to $23 \mathrm{~mm}$ in diameter, specifically dedicated to the study of the anal canal. This material provides excellent quality images and largely contributes to the development of $A E S$. The frequency used in the current practice varies from 5 to $10 \mathrm{MHz}$. With a $7.5 \mathrm{MHz}$ probe, the depth of field is around $5 \mathrm{~cm}$ and the axial resolution power in the order of a millimetre [3].

\section{EXAMINATION PROCEDURE}

The examination is performed without hospitalization after an evacuating rectal lavage. The patient is set in a gynaecological position or in lateral decumbency. Sedation is most often useless. First, it is better to perform a cursory proctologic examination in order to establish a relationship between clinical and ultrasound findings and therefore to avoid a risk for discrepancies in the final record. The images are progressively studied while the probe is taken out, and they can be printed and/or recorded. The technique is simple and fast to be performed (around 10 to 15 minutes), cost effective (K30 in the French medical health quotation system) and without secondary effects [3].

\section{PATHOLOGICAL IMAGES}

AES allows for the topographical evaluation of anal fistulae (Table I) (Figures 1 to 6) and allows for the appraisal of the sphincter apparatus :

\section{Orifice opening (photograph 1)}

It is found as a heterogeneous zone located at the level of the first hyperechogenous layer (mucosa) 
TABLE I

SIMPLIFIED CLASSIFICATION OF FISTULA IN ANO ACCORDING TO LUNNISS ET AL. [44]

\begin{tabular}{|l|}
\hline Openings: \\
\hline - primary (anal crypts, rectal ulcers etc.) \\
- secondary (skin of the buttock of anal margin, rectal or vulvo- \\
vaginal mucosa) \\
\hline Main fistulous tracks: \\
\hline - inter-sphincterian \\
- trans-sphincterian \\
- supra-sphincterian \\
- extra-sphincterian \\
\hline Secondary extensions: \\
\hline - intra-mural \\
- supra-levatorian \\
- horse shoe shaped (through the ischio-anal, inter-sphincterian \\
or supra-levatorian space) \\
\hline Collections: \\
\hline - inter-sphincterian \\
- intra-mural \\
- ischio-anal \\
- supra-levatorian \\
\hline
\end{tabular}

and/or at the level of the second hypoechogenous layer (internal sphincter) [4]. Possible air bubbles giving rise to hyperechogenous images with a typical posterior shadow cone are easily identified and facilitate the localization [5-11]. It is difficult, however, to say for sure that the primary opening is located within an anal crypt since the pectineal line cannot be visualized with $A E S$.

\section{Main fistulous track and possible secondary extensions (photographs 2 to 7 )}

The fistulous tracks are in general easy to see and bear the aspect of a hypoechogenous, ill-defined band, sometimes centred by air bubbles, most often located between the internal and external sphincters (intrasphincterian track) or prolonging itself through the external sphincter towards the ischio-anal fossa (transsphincterian track) $[5,6,8-11,13-15]$. The thickness of the tracks is greater if the examination is performed during an inflammatory phase due to the hypoechogenous aspect of pus when under pressure and/or because of the fistulous oedema. The extra-sphincterian tracks (those not involving the sphincter apparatus) are more difficult to identify because they are located at a distance from the exploratory field of the probe [6].

The anorecto-vaginal trans-sphincterian fistulae cause a specific problem. The ano-vaginal tracks are in general well visualized, all the most if they contain air bubbles and/or if they are inflamed. In contrast, the recto-vaginal tracks are difficult to identify, likely due to their rather short length [16-21].

\section{Collections (photographs 8 to 17)}

Their shape is extremely variable and their echogenicity varies upon their content. The purulent collections, very inflammatory ones, are in general frankly hypoechogenous and ill-defined due to the peripheral oedema. The chronic collections, little or not inflammatory ones, containing non-purulent granules and necrosis, are heterogeneous and better delineated than the former [5-8, 13-15]. These chronic collections could be difficult to distinguish from scar tissues, particularly common in multi-operated patients and/or within the scope of Crohn's disease, and this defines the main limitation of $A E S[5,6]$.

The ultrasound aspect also varies according to the anatomical spaces where the suppuration has diffused. The inter-sphincterian and/or intra-mural ones are particularly easy to visualize by AES. In contrast, some deep ischio-anal, or mostly supra-levatorian collections can be difficult to visualize because they are distant from the probe $[5,6]$.

\section{Defects of the anal sphincter apparatus (photograph 18)}

AES allows for the study of the sphincter apparatus and for the evaluation of possible defects. Such defects are frequent in the case of multi-operated fistula. The sensitivity and specificity of $A E S$, in this application, are close to $100 \%$ [22-25]. We will not develop this indication further than that we already reported $[2,3,26]$.

\section{IMPROVEMENT OF THE PERFORMANCES OF ULTRASOUND}

In a general way, the technique provides clearer images when the suppuration is active (presence of pus under pressure and oedema). A non contributive examination during a quiescent phase with intermittent anal fistula must therefore be repeated during an active phase, at the time of pain relapse [27-29].

It is also possible to enhance the pathological ultrasound images by injecting air, or better, hydrogen peroxide in the fistulous track through the secondary opening(s), if they are present [30]. The tracks and collections, hypoechogenous at first, thus become hyperechogenous and easier to visualize [4, 9-11, 20, 21,31-36]. Other contrasting dyes are being tested [37].

Some operators recommend to complete the endoanal examination with an endo-vaginal procedure [38]. If the probe allows to do so, it may be useful to use different frequencies. For example, a $10 \mathrm{MHz}$ frequency is more suitable for the identification of the primary opening, while $5 \mathrm{MHz}$ are better for the exploration of an extra-sphincterian track and/or a supralevatorian collection.

The precise location of the distance of the primary opening from the anus is difficult from the transversal images obtained with the rotative axial probes. The sectorial linear probes, which provide longitudinal images, are thought to be more informative. In addition, these latter probes are likely to be more useful to follow the fistulous tracks over their total length [39]. 
Tri-dimensional AES is still recent and the available data are scarce. One can predict, however, that it will contribute to the imaging of complex fistulous tracks $[12,40]$.

Last, in hyperalgic patients, the possibility to move the ultrasound machine allows to perform the examination in the operating room, under anaesthesia, just before operating.

\section{VALUE COMPARED TO OTHER EXPLORATION PROCEDURES}

AES is more valuable than the clinical examination under anaesthesia $[6,8]$ and computed tomography $[14,15]$ in the topographical evaluation of anal suppurations.

On the other hand, it is complementary of magnetic resonance imaging (MRI) [12]. AES is indeed easier to access to, simpler to perform and more cost-effective. Furthermore, it is thought to be more efficient for the identification of primary openings and inter-sphincterian and/or intra-mural collections [27, 39, 44-47]. Even though, MRI provides excellent quality images due to its good contrast resolution, to the possibility to perform sections in all the spatial planes, thus providing a 3-D analysis very interesting in the case of complex suppurations, and also thanks to its excellent distant analysis of the peri-anorectal cellular spaces, allowing for an easy identification of ischio-anal and/or supra-levatorian collections. MRI can also discriminate between scar tissues and collections, which is an important limitation of AES [5, 6, 27, 44, 48]. Last, it should be improved in the years to come due to the numerous possible sequences $[2,12,49,50]$. One should emphasize, however, that even though AES is an operator-dependant technique, $M R I$ requires a specific competence for its manipulation and interpretation, so that the choice of a physician with an excellent knowledge of ano-rectal anatomy and anal suppurations is absolutely essential [29].

Last, AES was not compared here to fistulography but this technique is now almost completely abandoned $[2,12,51]$.

\section{INDICATIONS}

Imaging is useless in the vast majority of fistulae for which a treatment is readily decided after clinical examination. However, AES can be useful in the following cases:

\section{Doubt for the diagnosis}

AES allows to document the differential diagnosis between a crypto-glandular fistula and a non-fistulous suppuration (bartholinitis, Verneuil's disease, infected pilonidal sinus, abscess, infected episiotomy wound, etc.). It is also especially valuable for the exploration of possible sub-fissurar suppuration. Last, in the case of unexplained pains, it may reveal a suppuration which had not been detected by clinical examination $[12,52]$.

\section{Complex suppuration}

A complex suppuration is characterized by it unusual anatomical extension (upper main track, multiple secondary extensions and/or deep collections), its tendency to relapse, its multiple surgical treatments, important scar remodelling, a subjacent anoperineal Crohn's disease, etc. An accurate topographical situation is therefore essential in order to optimize the efficacy of the treatment. A mistake in the identification of the primary opening or the ignorance of a deep collection and/or of secondary extension could in fact result in the lack of cure and/or a relapse. In this indication, AES should ideally be performed together with MRI $[2,12,29,52,53]$.

\section{Fear of de novo or aggravated post-operative anal incontinence}

The discovery of sphincter defect(s) has a medical and legal value, in particular in multi-operated patients. In addition, it can bring to favour to spare the sphincter (prolonged draining with a loose seton, lowering of a covering flap over the primary opening, injection of biological glue and/or anti-TNF alpha in the case of Crohn's disease) rather than a classical fistulotomy that could alter anal continence $[9,12,29$, 54].

\section{CONCLUSION}

Recently brought to use, AES imposed itself as a standard complementary exploration technique in proctology. It contributed in particular to improve the therapeutic management of suppurations because it provides a reliable topographical evaluation of the lesions and simultaneously allows for the assessment of the sphincter apparatus. However, an additional $M R I$ procedure can sometimes prove to be useful to complement its limitation, especially in the case of complex suppurations. 